Portland State University

PDXScholar

January 2011

\title{
Dark current in an active pixel complementary metal- oxide-semiconductor sensor
}

Justin Charles Dunlap

Portland State University

William Christian Porter

Erik Bodegom

Portland State University

Ralf Widenhorn

Portland State University

Follow this and additional works at: https://pdxscholar.library.pdx.edu/phy_fac

Part of the Physics Commons

Let us know how access to this document benefits you.

Citation Details

Justin C. Dunlap, William C. Porter, Erik Bodegom and Ralf Widenhorn "Dark current in an active pixel complementary metal-oxide-semiconductor sensor", J. Electron. Imaging. 20(1), 013005 (2011).

This Article is brought to you for free and open access. It has been accepted for inclusion in Physics Faculty Publications and Presentations by an authorized administrator of PDXScholar. Please contact us if we can make this document more accessible: pdxscholar@pdx.edu. 


\title{
Dark current in an active pixel complementary metal-oxide-semiconductor sensor
}

\author{
Justin C. Dunlap \\ William C. Porter \\ Erik Bodegom \\ Ralf Widenhorn \\ Portland State University \\ Portland, Oregon 97207
}

\begin{abstract}
We present an analysis of dark current from a complementary metal-oxide-semiconductor (CMOS) active pixels sensor with global shutter. The presence of two sources of dark current, one within the collection area of the pixel and another within the sense node, present complications to correction of the dark current. The two sources are shown to generate unique and characteristic dark current behavior with respect to varying exposure time, temperature, and/or frame rate. In particular, a pixel with storage time in the sense node will show a dark current dependence on frame rate and the appearance of being a "stuck pixel" with values independent of exposure time. On the other hand, a pixel with an impurity located within the collection area will show no frame rate dependence, but rather a linear dependence on exposure time. A method of computing dark frames based on past dark current behavior of the sensor is presented and shown to intrinsically compensate for the two different and unique sources. In addition, dark frames requiring subtraction of negative values, arising from the option to modify the bias offset, are shown to be appropriate and possible using the computational method. () 2011 SPIE and IS\&T. [DOI: 10.1117/1.3533328]
\end{abstract}

\section{Introduction}

Dark current is a major source of noise in digital imagers. To decrease the generation of dark current, many camera systems are cooled. In some cases, e.g. consumer cameras, a cooling system is not feasible and dark current can become a problem even for short exposures. A standard method for dark current correction is to take a so-called dark frame, an exposure with a closed shutter, either right before or right after the light exposure. This dark frame is subsequently subtracted from the actual image. Previously, ${ }^{1}$ we reported on a method to compute the dark frame for a CCD imager from the actual image which we want to correct for the dark current. This method has the following advantages: correction can be carried out without knowledge of the temperature and without loss of imager time to collect a dark frame. It will lead to improved image quality and will benefit image fidelity. Moreover, it can be done ex post facto and provides the ability to remove sensor specific information. ${ }^{2}$ It has been surmised that such computations should be feasible for CMOS imagers as well. With the rise of CMOS sensors' importance in consumer electronics, automotive applications, ${ }^{3}$

Paper 10038PRR received Mar. 8, 2010; revised manuscript received Dec. 2, 2010; accepted for publication Dec. 6, 2010; published online Jan. 12, 2011 .

1017-9909/2011/20(1)/013005/8/\$25.00 @ 2011 SPIE and IS\&T and security, ${ }^{2}$ and with the improvement of CMOS quality, this technique could be broadly applicable. The goal of the present study is to verify the applicability of the image correction algorithm, as previously applied to scientific CCD imagers, to a commercially available CMOS sensor.

The description of dark current in a CCD is similar to the analysis of dark current in a diode and requires taking into account several sources of dark current. ${ }^{4,5}$ We showed that a model of two exponential functions describes the dark current for the back-illuminated chip accurately. ${ }^{6}$ The knowledge of how each individual pixel's dark current changes with temperature can be used to calculate artificial dark frames. However, for many cameras the exact chip temperature is not precisely known. Our proposed dark current correction method requires no knowledge of the chip temperature or the camera settings. For instance, for a given exposure time, gain, and bias, the dark current of every pixel is characteristic of a specific temperature. The dark current of any pixel can therefore be used as an indicator of the dark count of all the other pixels on the chip. Impurities in the silicon cause some pixels to have an unusual dark count. ${ }^{7,8}$ These hot pixels have the highest signal-to-noise ratio and are the best temperature indicators. The basic idea is to use the dark current of hot pixels to sense the chip's state and predict the dark current of all pixels on the chip. Our previous work applied this method to several well-behaved scientific grade $\mathrm{CCD}$ imagers. The question remains whether the same or similar method can be applied to CMOS sensors.

Addressing noise in CMOS imagers has a long history, e.g., various hardware designs to minimize noise ${ }^{9,10}$ and modeling based on circuit models. ${ }^{11,12}$ Others have identified different sources of dark current. ${ }^{13}$ The present imaging performance of color CMOS sensors was reported to be inferior compared for high-end CCD sensors due to excessive dark current non-uniformity, conversion gain non-uniformity, image lag, pixel cross-talk, and low quantum efficiency. ${ }^{14}$ Further insights have been obtained by simulations of noise. ${ }^{15,16}$ Techniques for dark current correction based on the dark count in pixels outside the optically active imager are also proposed. ${ }^{17}$ In that work, these dark pixels are used as a proxy for the temperature of the chip and the dark current for the whole imager is extrapolated from it. In general, after dark current corrections are applied, all the subsequent image processing can be performed. ${ }^{18}$ 
First, we will present the protocol for dark current correction. The dark current data and its analysis are presented in the next section. Finally, we describe the results of the image correction on a flat field and show that there is a potential new twist that can extend the range of applicability of the image correction protocol.

\section{Dark Current Protocol}

In order to see what is involved in dark frame computation, we briefly summarize the correction protocol. ${ }^{1}$ A set of dark frames at various temperatures is taken, and the following basic steps are executed. The first step is to locate hot pixels to serve as temperature indicators. These are selected from an image, or images, with sufficiently large dark signal. A hot pixel in this context has a large dark signal compared to its neighboring pixels. The neighboring pixels are of significance because in an actual image containing light information, they are used to predict the light signal. Removing the light signal works accurately only if there is high degree of correlation between the light signal of the hot pixel and its adjacent pixels. In most images this correlation between adjacent pixels is very high. The hottest pixels are found by ranking the values of the pixels on the imager using the following equation:

hotpix $(x, y)=\sum_{i=-1}^{1} \sum_{j=-1}^{1}$ weight $(i, j) \cdot \operatorname{pixel}(x+i, y+j)$,

where $(x, y)$ are the coordinates of the pixel and pixel $(x, y)$ is its dark count. Only the immediate neighbors of the hot pixel are considered. However, it is possible to include a wider area and increase the range of the summation. The weight $(i, j)$ depends on the relative location of an adjacent pixel. Various weighting factors can be chosen. The sum of all the weighting factors equals to zero, such that if all nine pixels are equally hot, hotpix $(x, y)$ is equal to zero. We chose the following weighting factors:

For the center pixel (the possible hot pixel): weight $(0,0)$ $=1$

For pixels on the corner of the hot pixel: weight $(-1$, $-1)=$ weight $(-1,1)=$ weight $(1,-1)=$ weight $(1,1)$ $=-0.05$

For pixels directly adjacent to the hot pixel: weight $(-1$, $0)=$ weight $(1,0)=$ weight $(0,-1)=$ weight $(0,1)$ $=-0.2$.

The directly adjacent pixels have a larger weight than the corner pixel because the correlation of the light signal should be the highest for those pixels $(-0.05$ and -0.2 were chosen for the sake of simplicity). Notice that with these weighting factors, the value for hotpix $(x, y)$ is equal or close to zero if all nine pixels have roughly the same dark current.

The next step is to calculate the average of $\operatorname{hotpix}(x, y)$ for the $n$ hottest pixels using frames at different levels of dark noise. This average is used as an indicator for the relative amount of the chip's overall dark current, $D_{\text {Ind }}$. The value of hotpix $(x, y)$ is then fitted to, for instance, a quadratic function of the indicator, $D_{\text {Ind, }}$ such that:

hotpix $(x, y)=a(x, y) \cdot D_{\text {ind }}^{2}+b(x, y) \cdot D_{i n d}+c(x, y)$.
Hot pixels which have a poor quality of fit are excluded. Next, the coordinates of all hot pixels, as well as the three fitting parameters, $a(x, y), b(x, y)$, and $c(x, y)$, are stored in a file which therefore contains all the information to evaluate the level of dark count of the chip. Note that one does not use the actual temperature, exposure time, bias, or gain (nor does one need to know these values). The calibration is a function of the dark count in selected dark current-indicator hot pixels.

Finally, it is necessary to determine the counts of all pixels with respect to $D_{\text {Ind }}$. To accomplish this counts of each pixel using frames with different levels of dark current are fitted with a quadratic least squares fit versus the dark current indicator. Since this fit is used later to calculate the dark count, independent from the neighboring pixels, the actual count of the pixels (not the signal with respect to the neighboring pixels) is used to determine the fitting parameters. The three fitting parameters can then be saved as images with the same dimensions as the chip. Once the imager is characterized and calibrated with these fitting parameters, dark frames can be calculated almost instantaneously over the whole calibrated range.

\section{Dark Current Measurements in the Firefly}

We used a Firefly MV camera with a 1/3" CMOS color sensor manufactured by Point Grey Research, Inc. to analyze the feasibility and the performance of such a dark current correcting algorithm for CMOS sensors. The chip is a double-buffered global-shutter photodiode device $(640 \times$ 480 pixels, $6.0 \times 6.0 \mu \mathrm{m}$ pixels, manufactured by Micron - MT9V022177ATC). The Firefly camera is well behaved in that after the temperature was stabilized for a sufficient time span, the measurements were repeatable. Automatic exposure control and color conversion were turned off. The camera was placed inside a temperature-controlled chamber.

The Firefly camera allows adjusting various parameters that are often not changed in a scientific CCD. The camera is capable of both 8 and 10 bit linear data acquisition. Presented in this work is exclusively 8 bit data, however the 10 bit data behaved similarly to the presented data. The gain could be set from 0 to $12 \mathrm{~dB}$ (or 2.8 DN/e to 11.4 DN/e) and was adjusted to optimally collect the data. The brightness setting, essentially a bias offset, can be adjusted such that pixels could potentially have no reading even after significant exposure times. In effect, these pixels have a negative offset. This has some interesting implications for dark frame correction and will be discussed in detail later. The camera could be set to frame rates of $7.5,15,30$, and $60 \mathrm{~Hz}$. The frame times used in this study, given as the inverse frame rate, are therefore given as 16.7, 33.3, 66.7, and $133.3 \mathrm{~ms}$. Exposure times could be set for each frame rate up to four times the nominal frame time. Therefore, apart from the maximum exposure time restriction, this camera allowed for independent adjustments of both the frame time and the exposure time. Both exposure time and the frame time were found to have an impact on the dark count.

All data was acquired by taking 10 frames and calculating the average while ignoring the two extremal data points.

First we investigated the dependence of the dark current on the exposure time, for times smaller and larger than the nominal frame time. The data presented in the two panels 

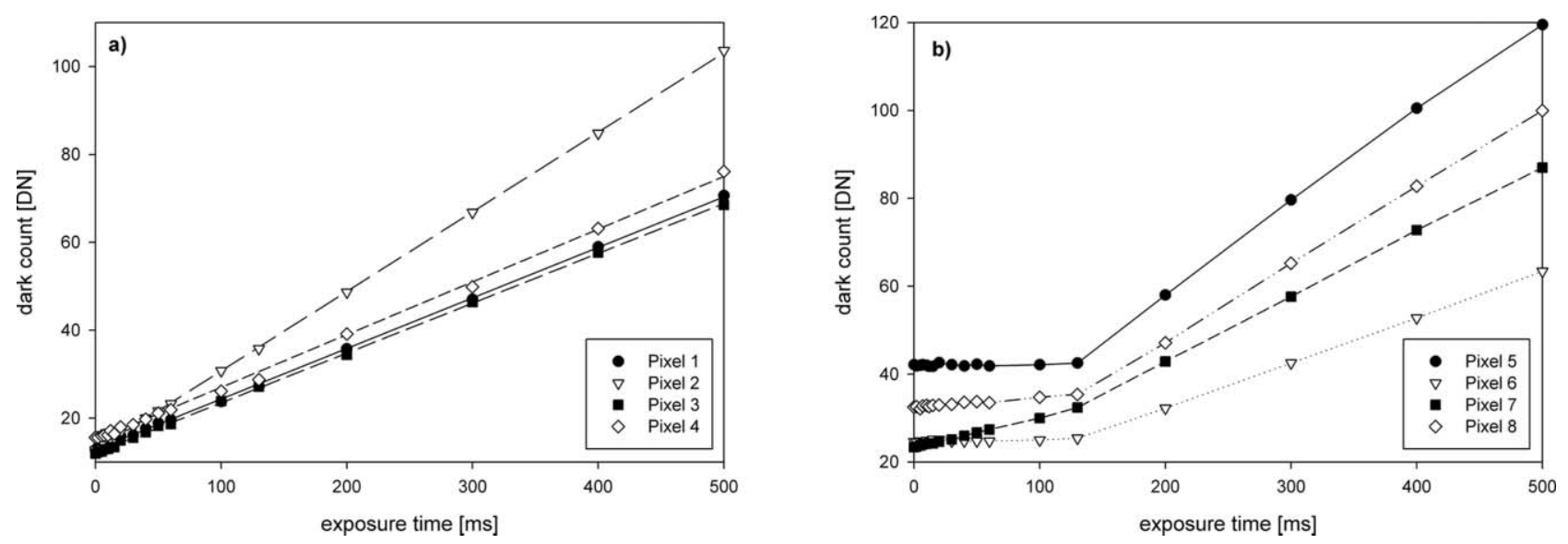

Fig. 1 Dark current vs. exposure time for individual pixels at $35^{\circ} \mathrm{C}, 0 \mathrm{~dB}$, and a frame time of $133.3 \mathrm{~ms}$. (a) With high dark current before the frame time $(133.3 \mathrm{~ms})$ and $(\mathrm{b})$ data with high dark current only after $133.3 \mathrm{~ms}$.

in Fig. 1 were taken at $35^{\circ} \mathrm{C}$ and a nominal frame time of 133.3 ms. Figure 1(a) shows a set of individual pixels, chosen to be representative of high dark current pixels for short exposure times less than $130 \mathrm{~ms}$. One finds that the dark count increases approximately linearly over the whole range of exposure times. For example, it can be gathered from the slope that Pixel 1 in Fig. 1(a) has dark current of about $0.18 \mathrm{DN} / \mathrm{ms}$, approximately eight times the average dark current at this temperature. Figure 1(b) presents pixels that have a large dark current at exposure times larger than the nominal frame time, but a smaller dark current for times less than $133 \mathrm{~ms}$. The behavior of these pixels is distinctively different. A distinct kink at the exact frame time can be observed. The dark count for three pixels in Fig. 1(b) is almost constant up to the frame time. This set of pixels, therefore, would have the appearance of being "stuck", or having a consistent level of dark current up to an exposure time that is the inverse of the frame rate. This type of pixel defect has been found in other CMOS sensors as an apparent time-independent offset. ${ }^{19}$ Pixel 7 shows a slight increase in dark count (0.067 DN/ms) for exposure time smaller than the frame

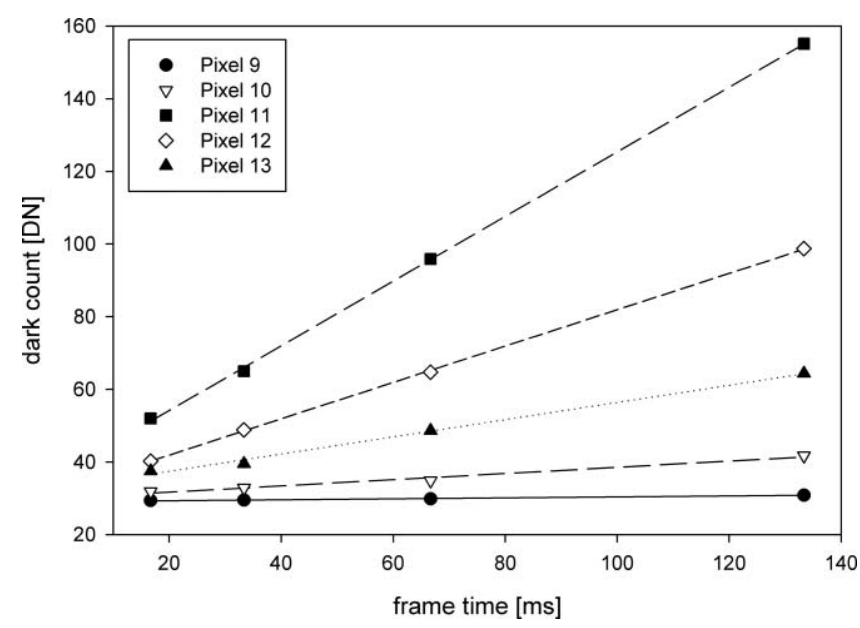

Fig. 2 Dark current versus frame time (1/frame rate) for selected pixels at $35^{\circ} \mathrm{C}, 12 \mathrm{~dB}$ with an exposure time of $10 \mathrm{~ms}$. time. For exposure times larger than the frame time, the dark current of all four pixels is seen to increase significantly. The slope of the dark count versus exposure time results in a dark current from $0.208 \mathrm{DN} / \mathrm{ms}$ for Pixel 5 to $0.102 \mathrm{DN} / \mathrm{ms}$ for Pixel 8.

To explain this change in slope, we will assume two distinct regions where dark current is generated. The dark current in the first region is visible for times shorter than the nominal frame time. This is presumed to be the dark current generated in the photodiode. For times larger than the nominal frame time, dark current produced from a second region becomes noticeable. Dark current in this region is generated for all exposure times. However, only for exposure time larger than the frame time does the measured dark signal increase with the exposure time. Unable to find out the exact configuration of the sensor from the manufacturer, we surmise that this location must be the floating diffusion, otherwise known as the sense node, used in the implementation of the global shutter mode. ${ }^{9}$ If the exposure time is larger than the frame time, the readout is delayed past the $133.3 \mathrm{~ms}$ and more dark current accumulates in the sense node. Up to the frame time, an impurity in this region contributes a constant amount to the total measured dark count. Other pixels that show a substantial dark current at times shorter than $133 \mathrm{~ms}$, show only a small effect when the exposure time is increased beyond $133 \mathrm{~ms}$, or in other words, the dark current in the sense node is small compared to that of the photodiode. However, a careful analysis reveals that almost all of the pixels in this chip have a slight kink at the nominal frame time revealing that most have some dark current generated in the sense node. Hence, it is necessary to differentiate between dark count generation in the photodiode and dark count generation in the sense node. In this CMOS sensor, one needs to keep track of two dark current generation rates.

To investigate the dark current generated in the two different regions, we varied the frame rate. In Fig. 2, the data for a $10 \mathrm{~ms}$ exposure at four different frame rates are plotted. Pixels were selected to give a representative sample of dark currents in the sense node. For example, for the same exposure time of $10 \mathrm{~ms}$, Pixel 9 in Fig. 2 produces a dark count of $52 \mathrm{DN}$ at a frame time of $16.7 \mathrm{~ms}$ and about 3 times as much (155.1 DN) at a frame time of $133.3 \mathrm{~ms}$. The 

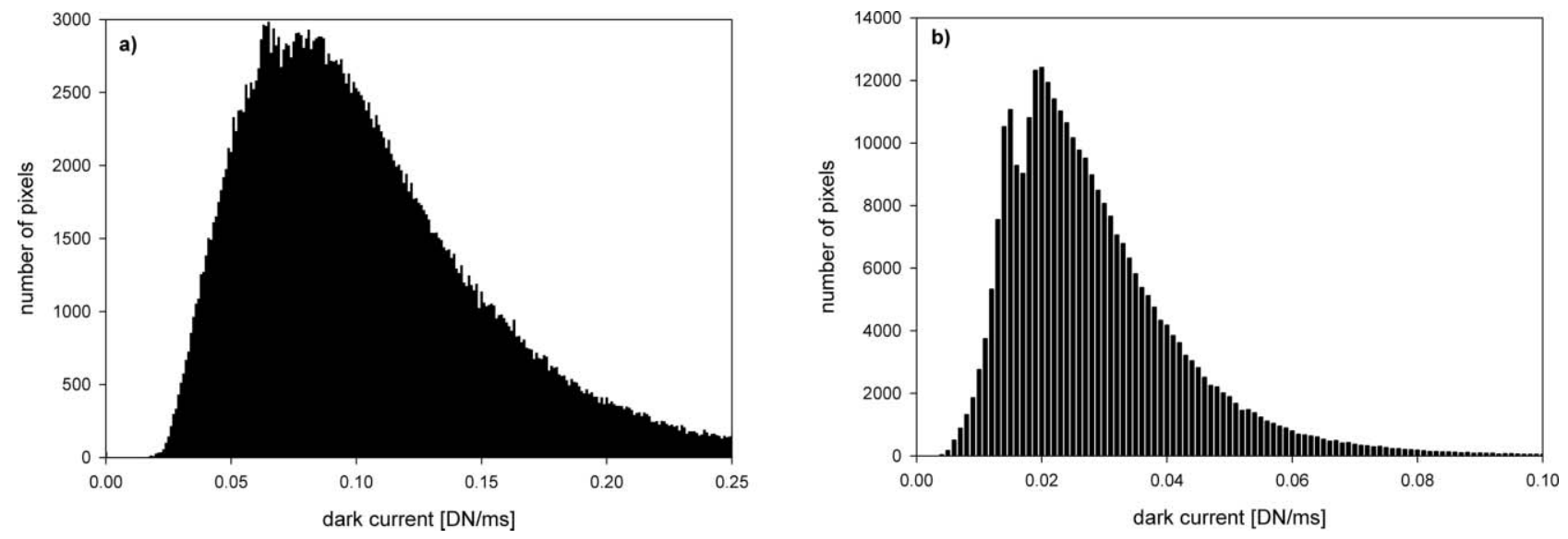

Fig. 3 Histograms of dark current based on exposure times longer than the frame time at $35^{\circ} \mathrm{C}$ : (a) with a gain of $12 \mathrm{~dB}$ and $(\mathrm{b})$ with a gain of $0 \mathrm{~dB}$ (both histograms binned in steps of $0.001 \mathrm{DN} / \mathrm{ms}$ ).

dark count of Pixel 13 is almost the same for all four frame rates. One would assume that the dark count collected in the photodiode of a particular pixel is the same for the different frame rates since the exposure time is fixed. The dark current accumulated in the sense node depends linearly on the frame time. As is borne out by the experiments, we see in Fig. 2 that the dark count indeed increases linearly with the frame time.

Figure 3(a) gives the distribution of the dark current at the largest gain $(12 \mathrm{~dB})$, based on a linear fit of the dark count versus exposure time, for times longer than the longest frame time $(133.3 \mathrm{~ms})$. The first observation is that there is a bimodal distribution. A clearer view of this bimodality shows up at the smaller gain. In Fig. 3(b), we present the data obtained for a gain of $0 \mathrm{~dB}$. The lesser gain serves to accentuate the two peaks. As a first estimate of the impact of the dark current one can find the curve fitted to the complete distribution. This distribution shows a peak at $0.08 \mathrm{DN} / \mathrm{ms}$ with a standard deviation of about $0.04 \mathrm{DN} / \mathrm{ms}$. Quite a few pixels will fill to $10 \%$ of full well in $100 \mathrm{~ms}$ (about $15 \%$ of the pixels) and another $30 \%$ will fill to $5 \%$ of full well in $100 \mathrm{~ms}$. It has been argued that in a typical camera, fixed pattern noise

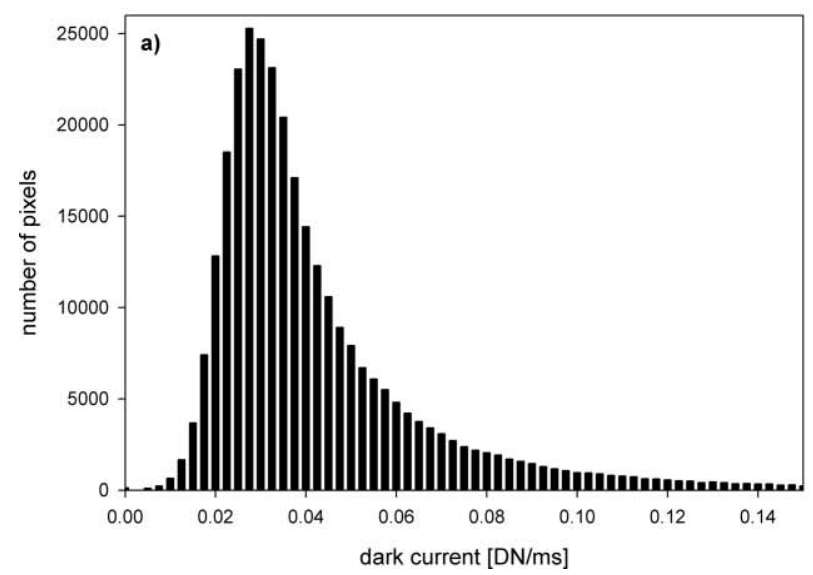

(FPN) should be less than $0.5 \%$ of full scale. ${ }^{10}$ From the distribution curve, we find that at $100 \mathrm{~ms}$ the FPN will not be acceptable for a consumer product. At higher temperatures, this will be aggravated even more.

In Fig. 4, we show the dark current distributions for exposure times less than the frame time, and of slopes for the dark counts vs. frame time. A log normal distribution gives a reasonable fit in agreement with the work reported by Baer. ${ }^{15}$ Referring back to Fig. 1(b) and the explanation of the kinks, i.e., the presence of two sources of dark current, one can infer the same from the two histograms. Figure 4(a) shows the dark current from the photodiode area and Fig. 4(b) presents the dark current contribution from the sense node. Hence, if one pairs the two independent sources, one would expect them to be additive. To a first approximation, the dark count contributions from the photodiode and the sense node are similar, i.e., they have the same mode around $0.03 \mathrm{DN} / \mathrm{ms}$. Since the peak contributions are the same, one would not expect to separate these individual peaks in Fig. 3, that is, if only one of the areas contributes, one would see a peak at $0.03 \mathrm{DN} / \mathrm{ms}$. If both contribute, another peak at more than $0.06 \mathrm{DN} / \mathrm{ms}$ should appear. The net result is that when both currents contribute,

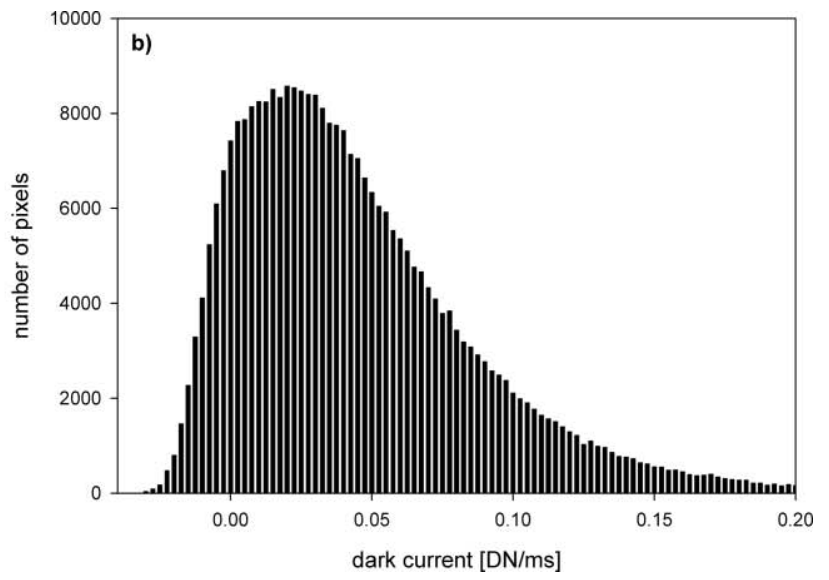

Fig. 4 (a) Histogram of dark current slopes $\left(35^{\circ} \mathrm{C}, 12 \mathrm{~dB}\right.$, frame time $\left.133.3 \mathrm{~ms}\right)$ for exposure time data less than the frame time. (b) Histogram of the slopes of dark count versus the frame times $\left(35^{\circ} \mathrm{C}\right.$, $12 \mathrm{~dB}$, exposure time $10 \mathrm{~ms}$ ). Both histograms binned in steps of $0.0025 \mathrm{DN} / \mathrm{ms}$. 

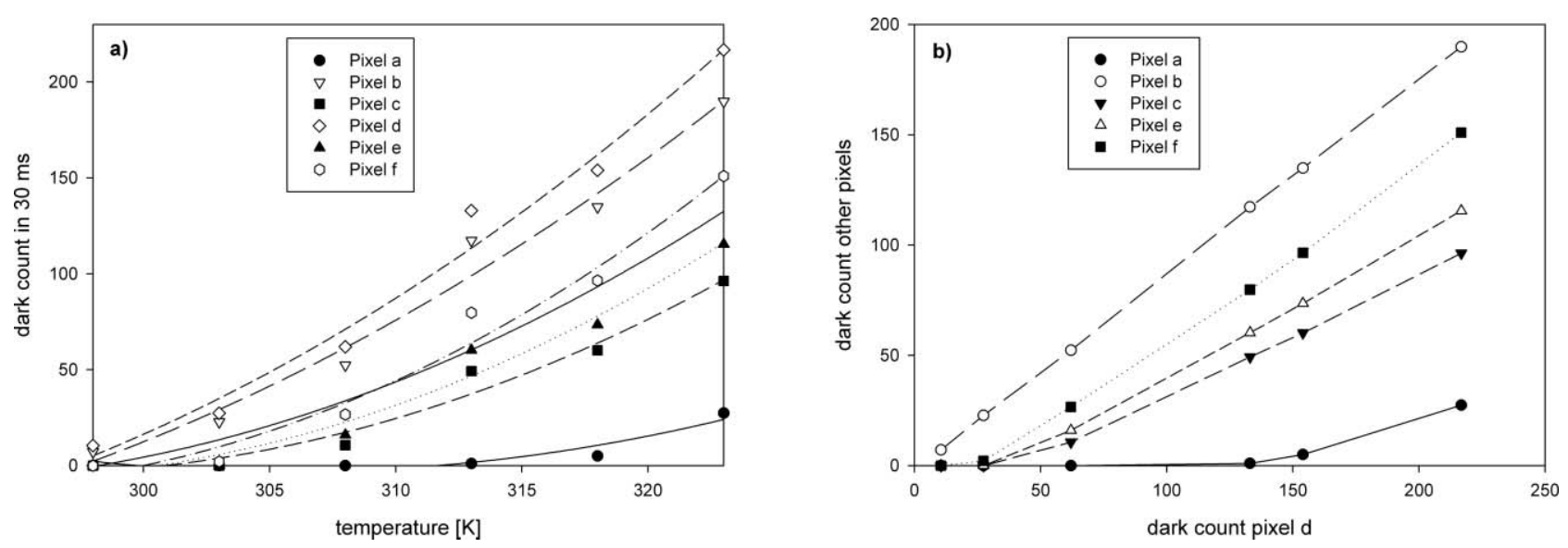

Fig. 5 (a) Dark current as function of temperature for several hot pixels (30 ms, $12 \mathrm{~dB}$, frame time $133.3 \mathrm{~ms}$ ). (b) dark count of Pixel a in Fig. 5(a) vs. the other pixels in Fig. 5(a).

another peak should appear in the distribution as is shown in Fig. 3.

After having analyzed the dark current of different pixels at different frame rates and exposure times, it remains to analyze their temperature dependence. Figure 5(a) shows the dark count of a group of randomly chosen hot pixels in a 30 $\mathrm{ms}$ exposure at various temperatures. The temperatures were measured external to the sensor and controlled to an accuracy of about $1 \mathrm{~K}$. The external temperature is not always an accurate representation of the chip's sensor. The sensor can be heated by operation of the chip and camera. ${ }^{20}$ For example, one sees in Fig. 5(a) that the dark count at $313 \mathrm{~K}$ is higher than expected from the other temperatures. Using hot pixels as an indicator, on the other hand, avoids the uncertainties of such temperature readings. The quadratic curve fits to the pixels show the overall tendency. Figure 5(b) shows the response of all pixels as a function of the dark count of Pixel a. One sees that the dark count of all pixels follow a well defined almost linear relationship. The dark count of Pixel a is a much better indicator of the temperature than the externally measured temperature. Hence, an indicator, calculated from the dark current of hot pixels, is a good proxy for the real temperature, which can then be used to predict the dark count of all pixels on the CMOS sensor.

It can be surmised from the data presented in Fig. 1 that dark noise is linearly dependent with exposure time before the frame time and is the sum of two linear contributions after the frame time. Therefore, dark current from the two sources can be described by the following empirical mathematical model:

for $t<t_{f}$ :

$D=D_{1} \cdot t$,

for $t>t_{f}$ :

$D=D_{1} \cdot t+D_{2} \cdot\left(t-t_{f}\right)$,

where $t$ is the exposure time, $t_{f}$ is the frame time, $D$ is the total amount of dark noise generated, and $D_{1}$ and $D_{2}$ are positive variables relating exposure time to the contributions of dark current from the collection area and sense node respectively.
In addition, as discussed in previous work, $D_{1}$ and $D_{2}$ vary as a function of temperature and can be assumed to follow the Arrhenius law:

$D_{1 \text { or } 2}=D_{1 \text { or } 2}^{0} \cdot \exp \left(-\Delta E_{1 \text { or } 2} / k T\right)$,

where $D_{1 \text { or } 2}^{0}$ are positive constants, $\Delta E_{1 \text { or } 2}^{0}$ are the activation energies, $k$ is Boltzmann's constant, and $T$ is the temperature. $^{6}$ If the observed dark current was generated in the depletion region, the dark current in electrons per second would be expected to be given as:

$D e_{\mathrm{dep}}^{-}=\frac{x_{\mathrm{dep}} A_{\mathrm{pix}} n_{i}}{2 \tau}$,

where $n_{i}$ is the intrinsic carrier concentration, $A_{\text {pix }}$ is the area of the pixel, $x_{\text {dep }}$ is the width of the depletion region, $\tau$ is the carrier lifetime. That is, it would be expected to increase linearly with $n_{i}$, which is given as:

$n_{i}=\sqrt{N_{v} N_{c}} \exp \left(-E_{g} / 2 k T\right)$,

with $E_{g}$ being the band gap in silicon, and $N_{v}$ and $N_{c}$ being the effective density of states for the valence band and conduction band respectively. ${ }^{6,11}$ However, if the dark current is not generated outside the depletion region it would be expected to follow the equation for diffusion current such that:

$D e_{\text {diff }}^{-}=\frac{D_{n} A_{\text {pix }} n_{i}^{2}}{x_{f f} N_{A}}$,

where $D e_{\text {diff }}^{-}$is now the dark current generated per second, $D_{n}$ is the diffusivity of electrons, $N_{A}$ is the acceptor concentration in the substrate, and $x_{f f}$ is the width of the field free region. Principally, now it is observed that the dark current increases as the square of the intrinsic carrier concentration. 6,21 Additionally, dark current generated on the interface of the silicon and silicon oxide layers, called surface dark current, should increase linearly with $n_{i}{ }^{21}$

When activation energies are calculated using the Arrhenius law, values close to the band gap of silicon are obtained regardless of whether it is calculated before or after the frame time suggesting that noise generated in both temporal regions may be diffusion current. 

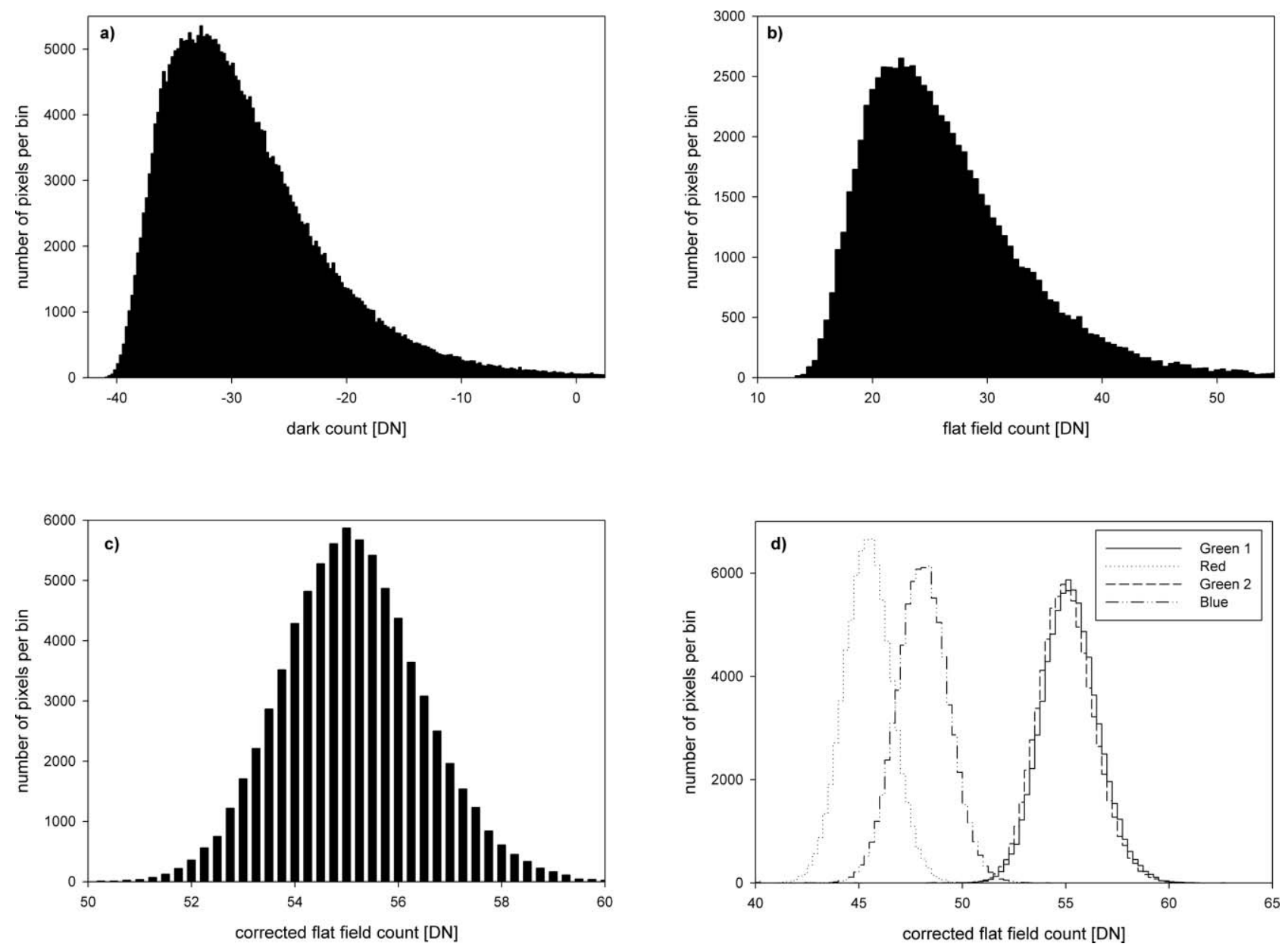

Fig. 6 Dark current correction of flat fields with a negative bias offset $\left(35^{\circ} \mathrm{C}\right.$, frame time $133.3 \mathrm{~ms}$ exposure time $50 \mathrm{~ms}$, and brightness = 1); (a) calculated dark frame distribution for the complete chip, (b) pixel values of half of the green pixels in the flat field, (c) the same green pixel flat minus the appropriate dark frame, (d) corrected flats of all pixels grouped by the Bayer array.

\section{Image Correction}

For any camera system the dynamic range is one of the most important, and possibly limiting, parameters. To utilize the maximum dynamic range one can adjust the bias offset depending on the illumination. For an image with a high enough signal it can be beneficial to adjust the bias offset to a negative number. That works without any loss of information as long as the light intensity is high enough to overcome the bias offset and cause a positive count for all pixels on the chip. For the Point Grey camera, the bias offset can be adjusted from about $-32 \mathrm{DN}$ for a brightness setting of 1, to $+37 \mathrm{DN}$ for a brightness setting of 255 . For example, with a brightness setting of 1 and a light intensity that would cause $3.2 \mathrm{DN} / \mathrm{ms}$, the response of a pixel would be zero up to an exposure time of $10 \mathrm{~ms}$. Only for exposures longer than 10 ms would the pixel actually give a nonzero reading.

The question we address here is: what happens to the dark frame when one takes an image with negative bias offsets? For many pixels, the dark current will be too small to reach $32 \mathrm{DN}$ for a given exposure time. For example the average dark current at $35{ }^{\circ} \mathrm{C}$ is equal to $0.075 \mathrm{DN} / \mathrm{ms}$ and most pixels will not exceed $32 \mathrm{DN}$ if the exposure time is less than $400 \mathrm{~ms}$. Therefore, a dark frame of, for example, $50 \mathrm{~ms}$ will display zero counts for an average pixel. The actual average dark count generated in $50 \mathrm{~ms}$ is still given by multiplying the dark current with the exposure time: $0.075 \mathrm{DN} / \mathrm{ms}$ times $50 \mathrm{~ms}=3.75 \mathrm{DN}$. For a hot pixel with a dark current of $0.5 \mathrm{DN} / \mathrm{ms}$, the dark count would be $25 \mathrm{DN}$, significantly higher than for an average pixel. However, both pixels would show up with a zero count in the dark frame. Therefore, to accurately correct for dark current, dark frames have to be obtained starting with a value equal to the bias offset, even if this might cause the somewhat surprising result of a dark frame with a large number of pixels that have a negative dark count. One can obtain such a dark frame by:

1. Calculating dark current as the count versus exposure time of an image with a large brightness setting.

2. Obtaining the correct bias offset from the y-intercept of a linear fit versus exposure time for light exposures with the correct brightness setting (in our case 1).

The dark count for brightness of 1 is then equal to the dark current in DN/ms (from step 1) times the exposure time plus the negative bias offset (from step 2).

Panel a in Fig. 6 shows the histogram of a dark frame calculated as described above at $35 \mathrm{C}$, exposure time of 
$50 \mathrm{~ms}$, frame time of $133.3 \mathrm{~ms}$, and a brightness setting of 1. The distribution, as is typical for a dark frame, is skewed to the right. Unlike a typical dark frame, the average value is negative $(-28.6 \mathrm{DN})$ and most of the other pixels have negative dark count values as well.

To test the dark current correction we obtained flat fields at the same setting as the dark frame. Because the Point Grey camera has a color chip with a Bayer RGB filter pattern, the individual color channels have to be analyzed individually. Panel $b$ shows pixel values of all green pixels (half of the pixels in the imager are green). One can see that the light intensity was high enough that all pixels reached a positive count. However, one would expect a Poisson like distribution for a flat field exposure. Due to the dark current, the distribution is skewed to the right. Subtracting a regular dark frame will not improve the situation, since most pixels will have a zero value. Using the dark frame obtained with the method described above, an accurate dark current correction can be done. Panel c of Fig. 6 shows the histogram for the flat field after subtraction of the accurate dark frame (see Panel a for the values of the dark frame). As one would expect, the distribution for the flat field is symmetrical with pixel values close to the average value. The average pixel value is given as $54.9 \mathrm{DN}$, larger than the average value before the dark current correction. The width of the distribution has decreased significantly. The standard deviation of 7.62 DN for the uncorrected frame has decreased to $1.39 \mathrm{DN}$ for the corrected frame.

Of course, the same correction can be applied to the red and blue pixels. Panel d shows the pixel values for all pixels. The first peak corresponds to pixels with a blue filter, the middle peak to red pixels, and the right peak is for pixels with a green filter.

CMOS architectures and geometries are known to be a factor in the amount of dark current generated. ${ }^{11,22}$ Future work should be done to analyze the dark current generated by different imagers and the effects on dark current correction, in particular the method of using hot pixels on the imager itself for correction. However, the arguments presented above show the robust nature of the method and its ability to accurately correct for dark current despite complications presented by modern CMOS sensors such as color filtered pixels, negative bias offsets, and multiple sources of dark current.

\section{Summary}

We have demonstrated that all the ingredients for a computed dark frame correction exist in a CMOS sensor. ${ }^{23}$ These ingredients include the presence of hot pixels that have a relatively large amount of dark current and therefore can serve as dark current indicators, and the pixels not chosen as indicators, demonstrate predictable dark current behavior. We have shown that a flat field corrected by a calculated dark frame produced a corrected flat field that is significantly better. Because there are two kinds of sources of dark current, one which depends solely on exposure time and the other which depends on frame time, it should, in principle, be possible to calculate temperature and exposure time. Indeed, the computational method does not require knowledge of the settings, and is successful for the correction of images even allowing for two regions of dark current production and a possible bias setting resulting in negative dark count values. Since the protocol allows for the correction of images for dark current over a range of settings and external variables, including temperature, it should prove useful in situations where the temperature of the sensor is elevated in, for instance, automotive applications.

\section{References}

1. R. Widenhorn, A. Rest, M. M. Blouke, R. L. Berry, and E. Bodegom, "Computation of Dark Frames in Digital Imagers," Proc. SPIE 6501, 650103 (2007).

2. J. Lukas, J. Fridrich, and M. Goljan, "Digital camera identification from sensor pattern noise," IEEE Trans. Information Forensics Security $\mathbf{1}$, 205 (2006).

3. B. J. Hosticka et al, "CMOS Imaging for Automotive Applications," IEEE Trans. Electron Dev. 50-1, 173 (2003).

4. A. S. Grove, Physics and Technology of Semiconductor Devices, Wiley, New York (1967).

5. S. M. Sze, Physics of Semiconductor Devices, second edition, Wiley, New York (1981)

6. R. Widenhorn, M. M. Blouke, A. Weber, A. Rest, and E. Bodegom, "Temperature dependence of dark current in a CCD," Proc. SPIE 4669 193 (2002).

7. W. C. McColgin, J. P. Lavine, and C. V. Stancampiano, "Probing metal defects in CCD image sensors," Mat. Res. Soc. Symp. Proc. 378, 713 (1995).

8. W. C. McColgin, J. P. Lavine, C. V. Stancampiano, and J. B. Russell, "Deep-level traps in CCD image sensors," Mat. Res. Soc. Symp. Proc. 510, 475 (1998).

9. G. Yang, O. Yadid-Pecht, C. Wrigley, and B. Pain, "A Snap-Shot CMOS Active Pixel Imager for Low-Noise," High-speed Imaging, IEDM 98, 45 (1998).

10. M. F. Snoeij, "Analog Signal Processing for CMOS Image Sensors," PhD Dissertation, TU Delft (2007).

11. B. Pain, T. Cunningham, B. Hancock, C. Wrigley, and C. Sun, "Excess Noise and Dark Current mechanism in CMOS Imagers," Proc. Int. Image Sensor Workshop 145, (2007).

12. H. Tian, B. Fowler, and A. El Gamal, "Analysis of Temporal Noise in CMOS Photodiode Active Pixel Sensor," IEEE J. Solid-State Circuits 36, 92 (2001).

13. H. In Kwon, I. M. Kang, B. G. Park, J. D. Lee, S. S. Park, "The Analysis of Dark Signals in the CMOS APS imagers from the Characterization of Test Structures," IEEE Trans. Electron Dev. 51, 178 (2004).

14. A. J. Blanksby and M. J. Loinasz, "Performance Analysis of a Color CMOS Photogate Image Sensor," IEEE Trans. Electron Dev. 47, 55 (2000).

15. R. L. Baer, "A Model for Dark Current Characterization and Simulation," Proc. SPIE 6501, 606805 (2007).

16. S. H. Lim, "Characterization of Noise in Digital Photographs for Image Processing," Proc. SPIE 6069, 606900 (2006).

17. R. L. Baer, "Efficient Dark Current Subtraction in an Image Sensor," US Patent 6, 714,241 (2004).

18. A. J. P. Theuwissen, "Image Processing Chain in Digital Still Cameras," VLSI Circuits, Digest of Technical Papers, 2 (2004).

19. J. Leung, G. H. Chapman, Y. H. Choi, R. Thomas, Z. Koren, I. Koren, "Analyzing the Impact of ISO on Digital Imager Defects with an Automated Defect Trace Algorithm," Proc. SPIE 7536, 75360F (2010).

20. J. C. Dunlap, E. Bodegom, and R. Widenhorn, "Correction of dark current in consumer cameras," J. Electron Imag. 19, 013010 (2010).

21. J. R. Janesick, Scientific Charge-Coupled Devices, SPIE Press, 2001.

22. I. Shcherback, A. Belenky, and O. Yadid-Pecht, "Empirical dark current modeling for complementary metal oxide semiconductor active pixel sensor," Opt. Eng. 41, 1216 (2002).

23. R. Widenhorn, A. Rest, R. L. Berry, and E. Bodegom, patent pending.

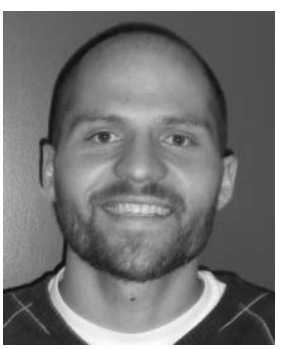

Justin C. Dunlap received his BA degree from the University of California, Berkeley in Physics and Astrophysics in 2002. He received his MS degree from Portland State University in Physics in 2009. He is currently working toward his $\mathrm{PhD}$ at Portland State University where he researches dark current and digital imagers while also working with undergraduate students to develop physics curriculum. 


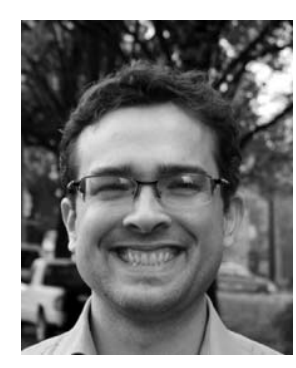

William C. Porter received his BS in physics from Portland State University in 2008, and is currently applying global and regional climate models to questions about aerosol and methane feedbacks as he works towards a $\mathrm{PhD}$.

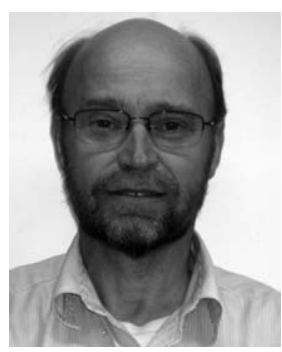

Erik Bodegom completed his engineering degree at Technical University Delft (Netherlands) and his PhD (1982) at Catholic University of America in Washington, DC. Since 1985 he has been with Portland State University, where he is a professor in the Department of Physics. His pedagogical activities have included the writing of a set of astronomical exercises, the development of a resistance probe for use in schools, and the development of software to remotely control observatories. He has served as President of the Oregon Academy of Science. His research activities have ranged from low temperature physics to statistical mechanics and solid-state physics. Over the last decade, his work has focused on the use and understanding of digital imagers.

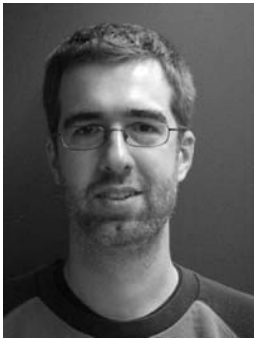

Ralf Widenhorn received his Vordiplom in physics from University of Konstanz in 1997, his MS in physics in 2000 and his Doctorate in environmental sciences and resources/physics in 2005 both from Portland State University. He has published numerous papers on digital imagers and is currently a researcher and professor at Portland State University. Some of his work is available in book from under the title Charge-coupled Devices Performance and Dark Noise Characteristics. 